\title{
A Bayesian Model for real-time safety management in construction sites
}

\author{
Emanuela Quaquero $^{{ }^{*}}{ }^{*}$, Carlo Argiolas ${ }^{1}$, Alessandro Carbonari ${ }^{2}$ and Filippo Melis ${ }^{1}$ \\ ${ }^{1}$ Università di Cagliari, DICAA Department, Cagliari, Italy \\ ${ }^{2}$ Università Politecnica delle Marche, DICEA Department, "Building Construction and Au- \\ tomation" Research Team, Ancona, Italy \\ *Corresponding author (equaquero@unica.it)
}

\begin{abstract}
Purpose This article reports on an ongoing research project, which is aimed at implementing advanced probabilistic models for real-time identification of hazardous events at construction sites. The model has intelligent capabilities for near real-time automated recognition of hazardous events during the execution phase. To achieve this, features of Bayesian Networks have been exploited. In addition, inputs to the model are assumed to be provided by a pervasive monitoring system deployed on the site. The need for this kind of intelligent tool is determined by the complexity inherent in construction sites, due to a variety of reasons, such as heterogeneity of the actors, the simultaneous nature of operations, harsh contextual conditions, and the only partially efficient current approach based on health and safety plans. Hence, this model is proposed as a support tool for health and safety coordinators for supervision of sites as they cannot guarantee a continuous physical presence. Method Given that there are no long-time series on past occurrences of hazardous events in all the potential contextual combinations presently available, the probabilistic models cannot be learned just through datasets. For that reason, the available data have been integrated with expert opinions. In particular, the conditional probabilities of the Bayesian networks are estimated by an elicitation process of subjective knowledge from the opinions of experts. The complexity of the phenomena under analysis are modelled as a tree structure with several levels (corresponding to the work-breakdown structure hierarchy), which itself is based on the top-down technique; it provides therefore a clear view of the global picture. The built-hierarchical tree allows the expert to weigh more easily causal relationships involved and also to define the qualitative structure of the net. Furthermore, the article describes and tests how conditional probabilities of the variables in the networks can be estimated, through gathering and interviewing groups of stakeholders and experts. Results \& Discussion Our research has led to the definition of a probabilistic model using elicitation techniques for subjective knowledge. Furthermore, the development of such a model is part of a wider system relying on the implementation of a real-time monitoring network.
\end{abstract}

Keywords: Automation, health and safety, Bayesian networks, construction sites.

\section{INTRODUCTION}

The building trade appears as a sequence of subprojects, rather difficult to manage owing to: the variety and the heterogeneity of the involved players, the independence and the simultaneous nature of related tasks, the singularity and complexity of the final product, the specific productive and contextual conditions. Such features, along with other elements, represent a strong barrier to the development of a rational planning approach, and they dramatically affect safety in construction sites, as shown by the many world statistics available in this field.

This paper reports the first results of an ongoing research project, which is aimed at implementing advanced probabilistic models for automatic real time identification of hazardous events in construction sites. More specifically, the development of a Bayesian model for real-time health and safety management is carried out, which will be subsequently interfaced to a sensing technology to gather contextual information.
In the next section, practices and relevant undergoing research in this field are described, spanning from construction site accident statistics to inabilities of the actual safety management system, and including a general report about research advances in the field of real-time risk analysis. The third and fourth sections focus on the development of the Bayesian model using the elicitation approach from the subjective knowledge and experience of safety experts. The fourth section includes some demonstrations of the developed model in the running phase, too. The last chapter concludes and envisages possible future scenarios for this research, mainly devoted to the model implementation.

\section{STATE OF THE ART}

\section{Construction site accident statistics}

The number of accidents on building sites will help understand the efficiency and the results by the application of the most popular models for safety management. 
A summary of national data on injuries at work shows that the construction industry is uppermost in the frequency of permanent disability or death. The number of accidents has decreased due to the economic crisis of recent years, along with the number of employed workers. The building sites are among the most dangerous workplaces and the number of accidents reaches up to $10-11 \%$ of the total reported in all manufacturing sectors. The number of accidents is higher in companies of handicraft character demonstrating the lack of organization and planning for health and safety ${ }^{1}$.

The number of fatal accidents in European countries (EU 15) is decreasing in these years (less than 3 fatalities per 100,000 employed) but in the construction industry the number exceeds 10 fatal accidents per 100,000 employed $^{2}$. The most frequent cause of accidents in the building industry is fall from height. The main causes are the execution of tasks without proper scaffolding, work on the roof, falls from unprotected openings or inadequate scaffolding. A recent study tested a number of incidents occurred in fall from height from different manufacturing sectors, highlighting how the percentage of fatalities in construction industry exceeds $57 \%$ of the total accidents. The same study reported that $91 \%$ of fatal falls took place in commitments where handicraft companies (fewer than nine employees) were involved. In the Italian context a recent study on the causes of accidents based on a number of 460 cases of fatal accidents in 2011 showed a percentage of $22,2 \%$ for the construction industry and, with respect to the causes, a percentage of $23,9 \%$ due to fall from height of the worker. The study do not indicate, however, the accident dynamics: the analysis were conducted in regional context by heterogeneous methods $^{1}$.

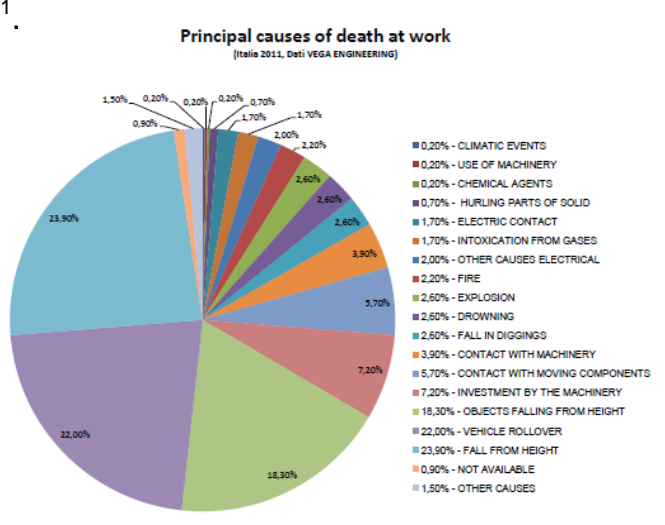

Fig.1.Principal causes of death at work (Italy, 2011)

In the U.S. construction industry one third of accidents are caused by fall from height. A study conducted by the Israeli Ministry of Labor and Welfare shows that $60 \%$ of accidents are due to fall from height and causes of these is in $41 \%$ by fall from slabs and roofs, in $19 \%$ fall from scaffolding and work surfaces, and $11 \%$ fall from ladders ${ }^{3}$.

Health and Safety management in construction Nowadays, the approach for health and safety management in construction industry is a standard practice in EU countries ${ }^{4}$ and it starts with the identification of tasks sequences at the design phase. Then, elementary working activities and preventive or protective actions are defined to safeguard workers. During the execution phase, an appointed Safety Coordinator is in charge of assuring the planned level of safety ${ }^{5}$. In USA, in recent years, the NIOSH (National Institute for Occupational Safety and Health) promoted the PtD strategy (prevention through design) as a standard that provides guidance on including prevention through design concepts within an occupational safety and health management system ${ }^{6,7}$. Thanks to the application of these prescriptions, decisions pertaining to occupational hazards and risks can be incorporated into the process of design and redesign of work premises, tools, equipment, machinery, substances, and work processes including their construction, manufacture, use, maintenance, and ultimate disposal or reuse. Both approaches (i.e. European and American standards) are based on the analysis of risk scenarios for each task and interfering activities ${ }^{8}$ expected in construction sites. Both models follow the PCDA cycle (Plan Check Do Act) ${ }^{9}$ :

- Step 1: hazard and risk identification;

- Step 2: classification of risks in order of priority;

- Step 3: definition of preventive and protective measures for all risks;

- Step 4: taking action to mitigate and reduce risks;

- Step 5: check and review of drawn up safety plan. The strength of the model lays in the ability to schedule the tasks time durations and the chance to reduce them to limit high-risk interferences. A weak aspect of the approach lays in the high costs tied to monitoring and control. Safety controls and checks, presently, imply strong, continuous physical presence of the Safety Coordinator on different construction sites in order to preserve the planned level of safety.

\section{Recent research and advances in real time risk analysis}

Statistics related to fatal/severe accidents on construction sites show that such places are still today the most dangerous ones. Besides causing human tragedy, construction accidents also delay project progress, increase costs, and damage the reputation of contractors. For this reason, a systematic control of operations is needed, which would however entail high costs defined "unsustainable" by some players. Safety controls and checks at present imply strong, continuous physical presence on the different work 
sites. There is an urgent need to develop methods allowing a cut-down in control costs through automatic alarm systems, warning hazardous situations potentially prone to degeneration into life and serious hazards, allowing for corrective operations in real time. A vast scientific literature regarding the development of technologies and methodologies to automatically support health and safety management is available. Even if supplying the complete list of works being performed in the field is a tough task, some of the most representative ones will be cited in this section. Instruments have been experimented on certain sites, different from construction sites because more structured with specific safety requirements (such as oil product refineries) which allow having real time knowledge of the position of workers present on the site. This has improved the situation involving interference between operators even if, in any case, only within the limits of surveillance delegated to an operator who is always present at the control boards ${ }^{10}$. Other systems for reducing risks regarding workers being run over by site equipment have been experimented as, for example, UWB radar systems installed on dumpers used in caves $^{11}$. Following another approach ${ }^{12}$ a 3D model is carried out starting from the data acquired by a LADAR (Laser Detection and Ranging) scanner so as to recreate the bulk of heavy objects present on the site and collision avoidance procedures. In this specific case, the approach was successfully validated but within the limits posed by the availability of an unobstructed visual filed, which is not always guaranteed on a construction site. Some years ago a compulsory safety helmet required for all workers in construction sites was enhanced to accommodate miniature positioning and communication instruments ${ }^{13}$. A different approach to safety is the substitution of human labor with machines to perform dangerous tasks: one example is given by the specially designed light-weight robotic tool, for the application of advanced composite materials and epoxy resins during tunnel excavation ${ }^{14}$. Another system against man-hook crane collisions was conceived ${ }^{15}$ : it starts from modeling workspace requirements related to mobile crane operations, and discerning the existence of spatial conflicts. Then it makes project engineers and operators aware of possible spatial conflicts ahead of time, so that they can accordingly take necessary preventive actions. A lot of effort was focused on fall hazards too. A mobile sensing device (i.e. transmitter sets and repeaters for sending information to a receiver) for detecting the worker's approach towards floor openings was proposed ${ }^{16}$. An automated procedures that identifies dangerous activities in the project's schedule at the design stage was set up; it also defines the areas in the building where these hazards appear and proposes protective measures ${ }^{3}$. The contribution given by ICT devices is critical for data gathering and immediate elaboration, but two pre-conditions have been recognized as critical for the success of ICT applied to safety: tracking systems must be low-intrusive and, also, they must be able to gather information in realtime, overcoming the limitations connected to manual information retrieval and handling, which has potentials for delays and errors. For that reason an ultra-wideband (UWB) wireless and untethered network system for mobile asset tracking at a dynamic construction site was tested $^{17}$. Its untethered nature favored the easy sensor nodes frequent relocation usually needed while work progresses. In addition, it avoids hazardous interferences between the network cables connecting all sensors on the floor or ceiling and crews engaged in material delivery and installation. The performances of this new UWB system were good, at the expense of a bit loss of accuracy, with respect to the basic tethered UWB system ${ }^{18}$. Combining non invasive tracking systems with dedicated intelligent control logics, would make the automation of many important tasks for construction sites feasible ${ }^{19}$.

\section{A PROBABILISTIC MODEL FOR REAL TIME RISK MAN- AGEMENT: A BAYESIAN NETWORK TO SUPPORT SAFE- TY IN CONSTRUCTION}

In this paper the progress status of our research, which is aimed at implementing advanced probabilistic models for real time identification of risk situations in construction sites, is reported.

To this purpose the features offered by Bayesian networks $^{20}$ are exploited. There are two main sources of information from which a Bayesian network can be developed: data and expert opinions. In this paper the Bayesian network was worked out by means of an elicitation process from the subjective knowledge of experts, in order to overcome the lack of data sets. As in the field of health and safety in construction there are no long time series on past occurrences of hazardous events in all the potential contextual combinations presently conceivable, the probabilistic models cannot be trained through just datasets. For that reason, the paper shows how the few available data have been integrated with expert opinions. The complexity of the problem under analysis are modelled as a multi-layered tree structure (corresponding to the Work Breakdown Structure hierarchy), which is based on the top-down technique; it provides therefore a clear view of the global picture. The built hierarchical tree allows the expert to weigh more easily causal relationships involved and to define the qualitative structure of the net, too. Furthermore, the article describes and tests how conditional probabilities of the variables in the network can be estimated, through gathering and interviewing groups of stakeholders and experts. 
In Fig.2, a flowchart is used to sums up all the process phases which led to the definition of the network described in this paper.

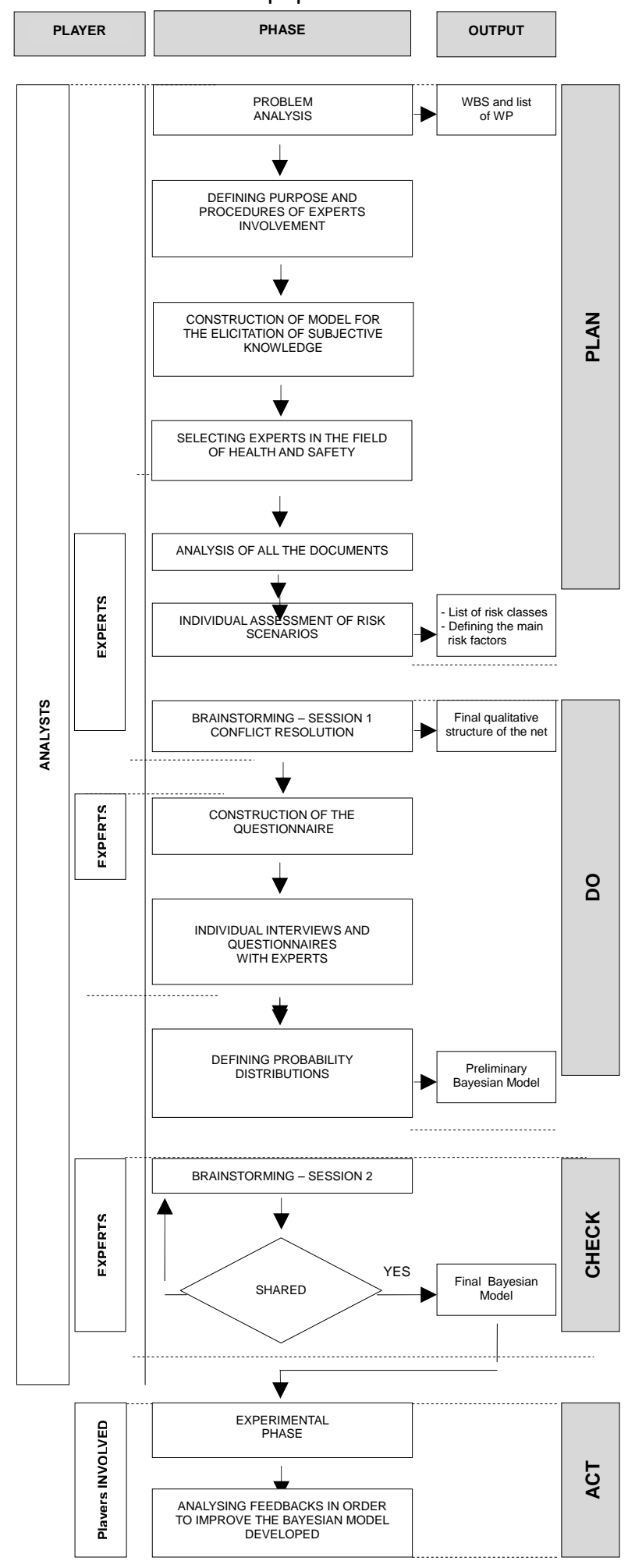

Fig.2.Construction and use of the Bayesian network

\section{Problem analysis}

De-structuring the building process: work breakdown structure

When the building process is represented as a tree structure with several levels (Work Breakdown Structure), following the top-down technique ${ }^{21}$, the outcome is a cascading representation, that defines all its elementary components in details (WPs, like in Fig. 3).

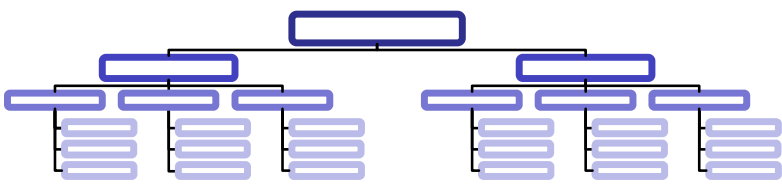

Fig.3. Tree-like decomposition (W.B.S.)

The development of the WBS and its efficacy in a process are straightforwardly affected by the level of accuracy used to identify all the components of the building process. As soon as the required level of appropriate accuracy is reached the decomposition process is over. It is worth noticing that the decomposition level varies according to the characteristics of the work to be carried out. In fact it is correct to say that the WBS can be divided into any number of levels, according to the intervention complexity, nevertheless, if the destructuring is extreme, it is difficult to keep track of the work progress, particularly if it has a long-term planning.

The built hierarchical tree allows an effective and deep understanding and control of the process regarding the safety aspects.

Following the analysis in detail of the building process and its de-structuring into elementary components, procedures and purpose of experts involvement have been defined. Furthermore, a model, which is aimed at gathering information from all the experts, has been developed. The model provides, to the experts, a guidance in eliciting process and, at the same time, it allows the analysts to gather homogeneous information.

\section{Elicitation process of subjective knowledge: expert opinion}

In this section of the paper, the elicitation process from subjective knowledge is shown.

\section{Selecting experts: session A}

In order to develop the Bayesian model, 6 experts have been selected. This selection led to set up a team with expertise in the field of safety management in construction sites. The table below shows the list of the experts involved and their specific contribution. 


\begin{tabular}{|l|l|l|}
\hline$N$. & \multicolumn{1}{|c|}{ EXPERT } & \multicolumn{1}{c|}{ CONTRIBUTION } \\
\hline 1 & $\begin{array}{l}\text { Professor in the field of construction } \\
\text { management }\end{array}$ & $\begin{array}{l}\text { His contribution to the research project } \\
\text { focuses on theoretical aspects }\end{array}$ \\
\hline 1 & Occupational medicine phisycian & $\begin{array}{l}\text { His contribution to the research project } \\
\text { focuses on aspects related to the } \\
\text { protection of workers in their } \\
\text { employment from risks resulting from } \\
\text { factors adverse to health }\end{array}$ \\
\hline 1 & $\begin{array}{l}\text { Occupational safety and health } \\
\text { inspector }\end{array}$ & $\begin{array}{l}\text { Will the aspects related to production } \\
\text { can affect their contributions }\end{array}$ \\
\hline 1 & $\begin{array}{l}\text { Health and safety coordinators about safety and } \\
\text { over } 10 \text { years of experience }\end{array}$ & $\begin{array}{l}\text { Established and detailed knowledge } \\
\text { about safety management in } \\
\text { construction sites }\end{array}$ \\
\hline 1 & Scaffolding fitters & \\
\hline
\end{tabular}

Fig.4. List of the experts involved

Following the selection phase, all the documents about the problem (WBS, WP and related information) have been gathered and circulated among the involved experts. The objectives of this step are to ensure that everyone involved has the same information pertinent to the problem.

\section{Defining causal model: session $B$}

Once all the documents have been analyzed, the experts were asked to specify all the activities which are necessary to create each defined elementary component (WP) and, for each activity, they have defined some parameters like machines, materials, equipment but also the labor required to carry out every task (Fig. 5). On the basis of these parameters the linked risks have been identified. The experts gave back this information using the model defined above.

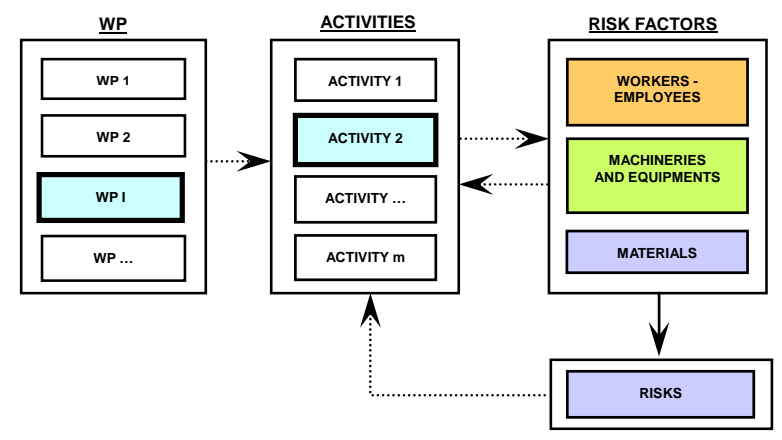

Fig.5. Relationships between WP, activities, workers, machineries, materials and risks.

By mean of a first general questionnaire, the experts were asked to reflect individually about each risk scenario identified previously. In this way each expert was given the time to think about the problem and to develop his opinion before debating with the other experts. The aim of this first questionnaire is to give to a quiet individual as much prominence as a dominating one.
The article showed, in the section "state of the art", that the most frequent cause of accidents, in construction sites, is fall from height (it represents almost $50 \%$ of fatalities). For that reason, our research project is focused on "fall from height risk" and the experts were asked to define the main dynamics about it. The outcomes of this phase were:

- fall from scaffolding,

- fall from carpentry/formworks,

- fall from roof.

Information and knowledge gathered in this first phase have been successfully summarized through "cause and effect" diagrams (see Fig. 6). These diagrams have been proposed during the following brainstorming phase as the tools for a structured discussion.

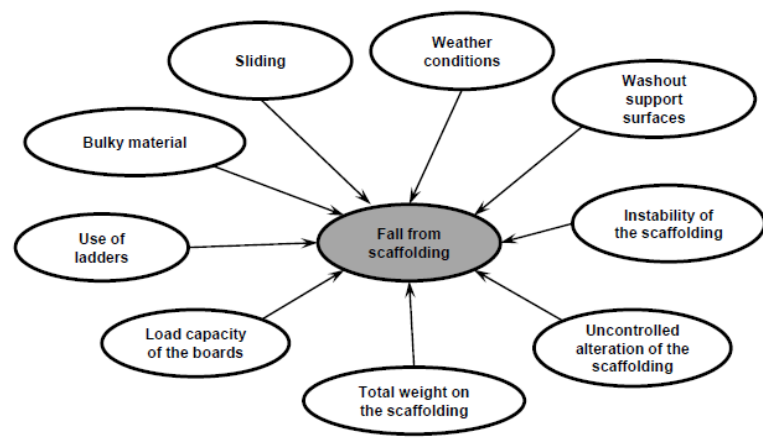

Fig.6. The main causes elicited about "fall from scaffolding"

In order to define each risk scenario and its main causes in detail, a specific questionnaire has been made "in itinere". Through the questionnaire the experts were conducted to develop the first qualitative structure of the Bayesian net. As opposed to the previous one, this questionnaire, which was developed in this phase, is aimed at structuring the brainstorming session in order to obtain a shared logical structure of the problem (Fig. 7). 


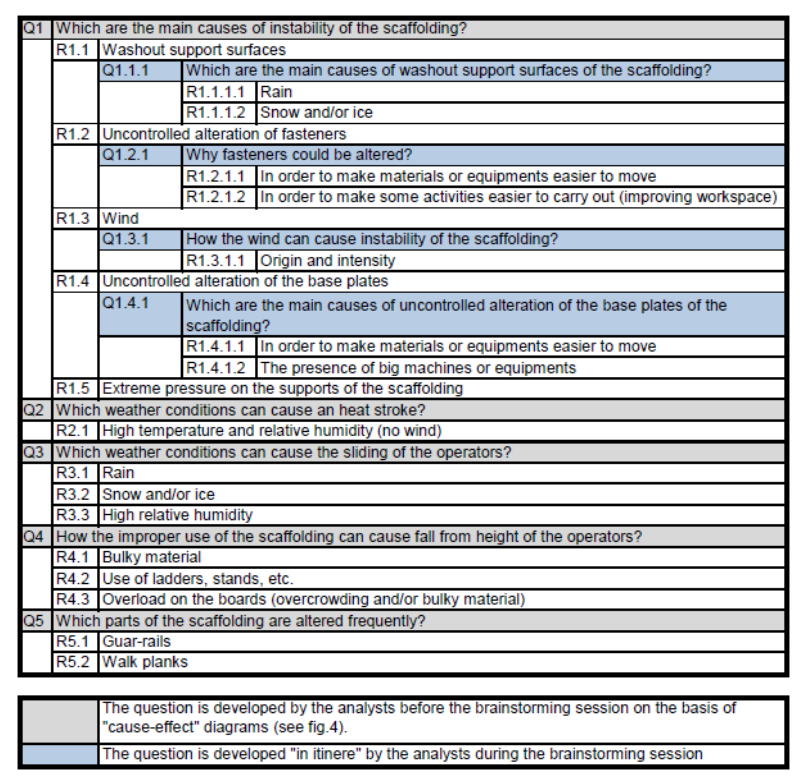

Fig.7. An excerpt from the questionnaire for the logical structuring of the problem.

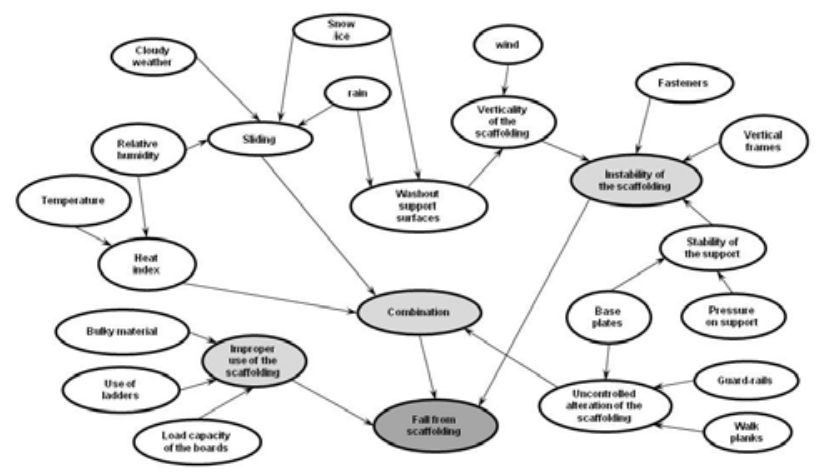

Fig.8. Fall from scaffolding: the qualitative BN structure

Other two sub-nets have been developed with the same procedure after the brainstorming session (see Figs. 9 and 10). These nets define two additional scenarios related to fall from height risk.

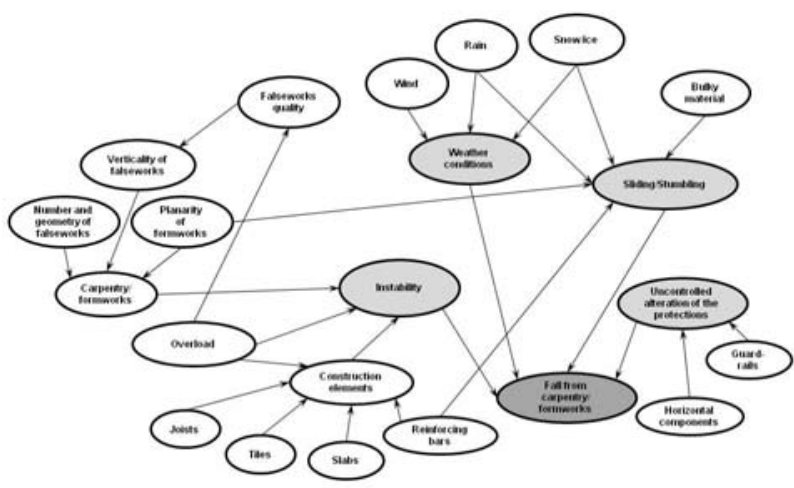

Fig.9. Fall from carpentry/formworks: the qualitative structure

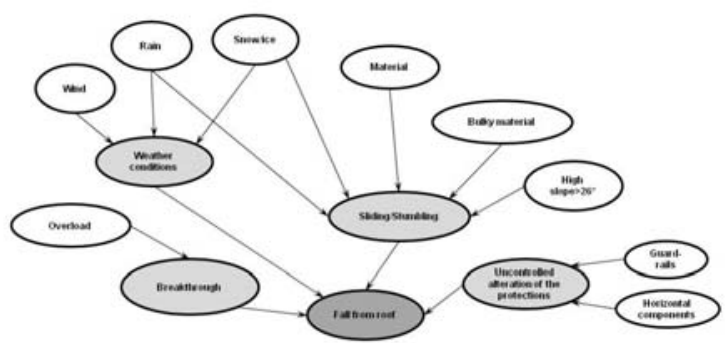

Fig.10. Fall from roof: the qualitative structure

Learning probability distribution for all uncertain variables of the model: session $C$

Expert opinions of the uncertainty of parameters has been determined through one-to-one interviews. After a suitable period for contemplation following the brainstorming session, we have carried on individual interviews with all the experts to determine their opinions about the qualitative structure of the model and the uncertainty in each variable. In this way each expert has been given the time to think about the problem and to develop his opinion after debating with the other experts.

Beginning from the qualitative structures of the net, the experts were asked to define, individually, a first information set related to:

- the states of all the network nodes;

- equations which allow to formalize links between variables in the model.

Information gathered in this phase have allowed us to create conditional probability tables for each part of the Bayesian network.

Beginning from the last node of the network "fall from scaffolding", the first fragment has been analyzed (see Fig. 11).

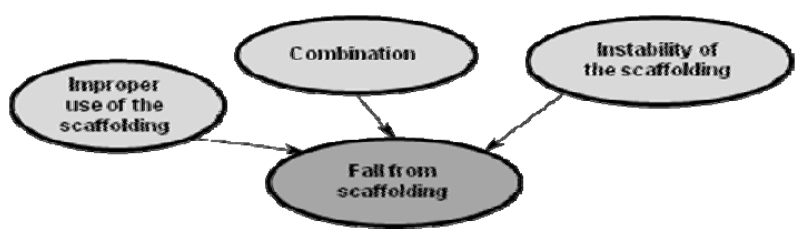

Fig.11. The first fragment of the network "Fall from scaffolding"

In order to develop this part of the network, the information about all the states of each variable has been gathered, coming to the structuring of the table of conditional probabilities. In this case, the experts have defined the states "low", "medium" and "high" for the variable "fall from scaffolding" and the states "not serious", "serious" and "extremely serious" both 
for the variable "Improper use of the scaffolding" and also for the variable "combination".

When the table related to this first fragment of network has been structured, we have defined a questionnaire which was aimed at gathering the expert opinions about probability distributions of the variable "fall from scaffolding".

Each expert was allowed to fill in the table of conditional probabilities on the basis of his/her personal experience.

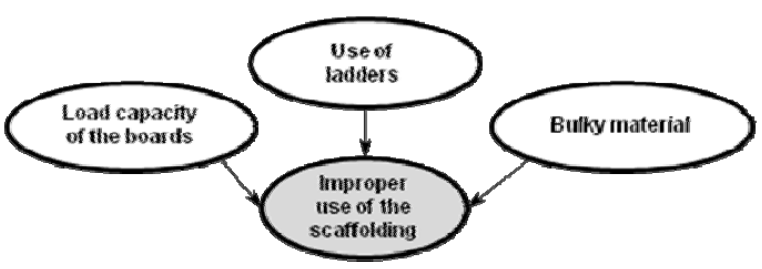

Fig.12. The second fragment of the network: "Improper use of the scaffolding"

The second fragment of the network is related to "Improper use of the scaffolding". In this case the experts defined the states "yes" and "no" both for the variable "Use of ladders" and for the variable "Load capacity of the board". Then, the experts have specified the states of the variable "bulky material". For this last variable, it was necessary to refer to the level of obstruction for the presence of bulky material on the scaffolding walk planks. In this case, expert opinions have led to define the following three states for the variable "bulky material":

State1: $0 \%<\mathrm{PO}<30 \%$ low obstacle: Caution

State2: $30 \% \leq \mathrm{PO}<60 \%$ medium obstacle: Danger State3: $60 \% \leq \mathrm{PO}<100 \%$ high obstacle: High danger When the table related to this second fragment of network has been structured, we have defined another questionnaire in order to gather the expert opinions about probability distributions of the variable "Improper use of the scaffolding" (see Figs. 13 and 14).

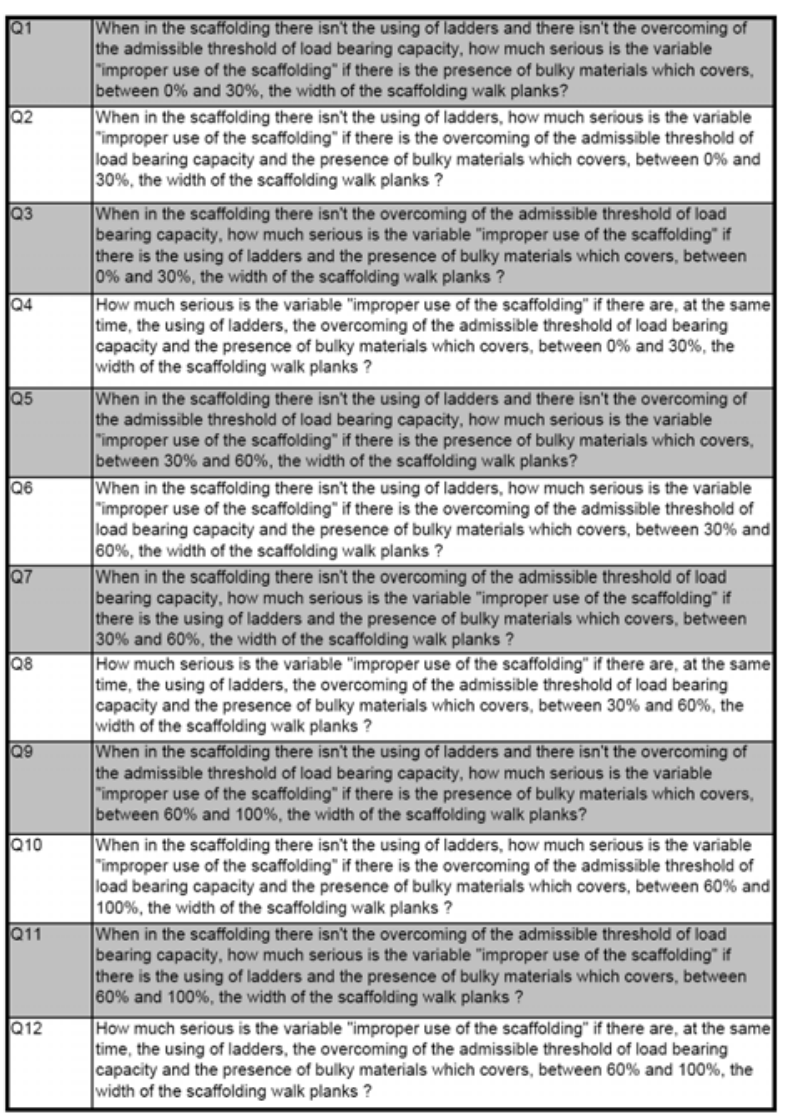

Fig.13. Questionnaire: probability distributions of the variable "Improper use of the scaffolding

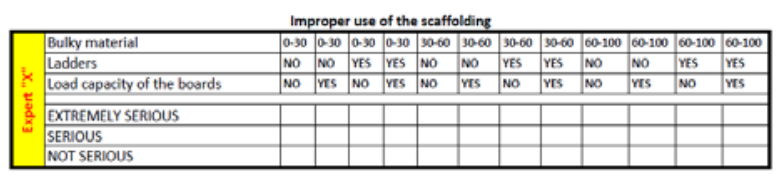

Fig.14. Table of conditional probabilities of the variable "Improper use of the scaffolding"

Sometimes we have obtained different estimates by the experts. This is usually because the experts made differing assumptions or have different sets of information on which their opinions are shaped (Fig. 15).

The paper shows how we have approached the problem.

We have used a Discrete $\left(x_{i}, p_{i}\right)$ distribution where the $x_{i}$ are the expert opinions and the $p_{i}$ are the weights given to each expert ${ }^{22}$.

In this way, once the tables of conditional probabilities of a specific variable have been gathered, we have combined dissimilar expert opinions and the result was obtained as a weighted table (Fig. 16).

The weights have been changed in relation to the specific part of the network. Each part of the net, in fact, requires different experience because it defines a particular aspect of the same problem.

With the same procedure all the other fragments of the network have been analysed, coming to the defining of a preliminary Bayesian model. 

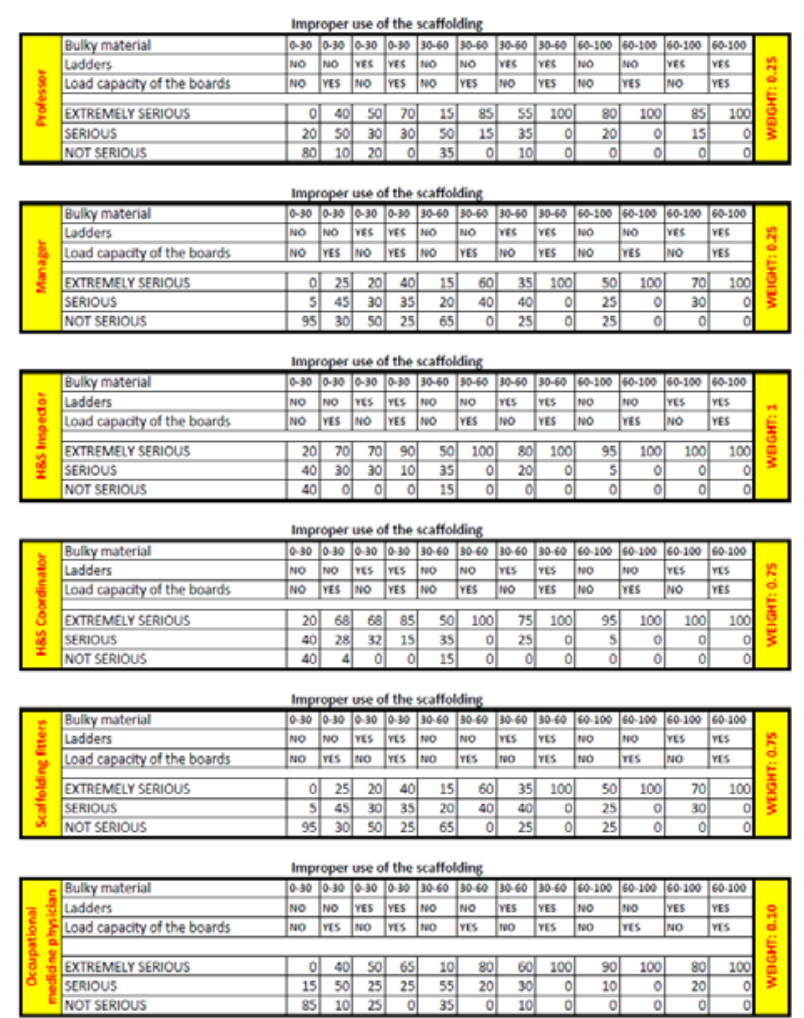

Fig.15. Dissimilar expert opinions

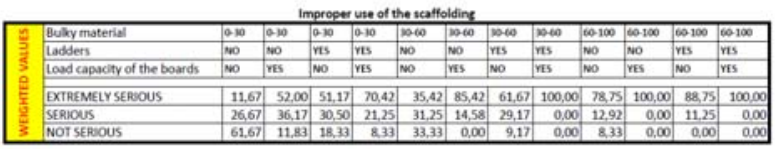

Fig.16. Combining dissimilar expert opinions: weighted table

In order to reach a concerted and definitive structure of the Bayesian model, another brainstorming session has been conducted. In this phase each expert has been given the last possibility to suggest changes to the Bayesian model.

\section{NETWORK IMPLEMENTATION}

Once the Bayesian network has been developed, it is ready to be implemented on a real construction site. Prior to this step, which belongs to future research, in the following sub-section we will show that the whole network is sensitive to the inputs relevant for the risk level estimation. The network was implemented in the Hugin Expert ${ }^{\mathrm{TM}}$ software program.

The second sub-section will assess on the feasibility of the network implementation for real-time warning in case of falling hazards: such an application asks for the availability of several kinds of input data, including on one hand the effective operational configuration of the site and on the other hand real-time inputs relative to workers behavior, weather conditions, etc.

\section{Preliminary tests}

The first scenario in Fig. 17 shows our network's risk evaluation in case the task activity is executed compliant to regulations and there are no adverse weather conditions. The risk level is not so high in this case $(<\mathrm{low}>=18.63 \%$, $<$ medium $>=23.39 \%$, $<$ high $>=57.98 \%)$.

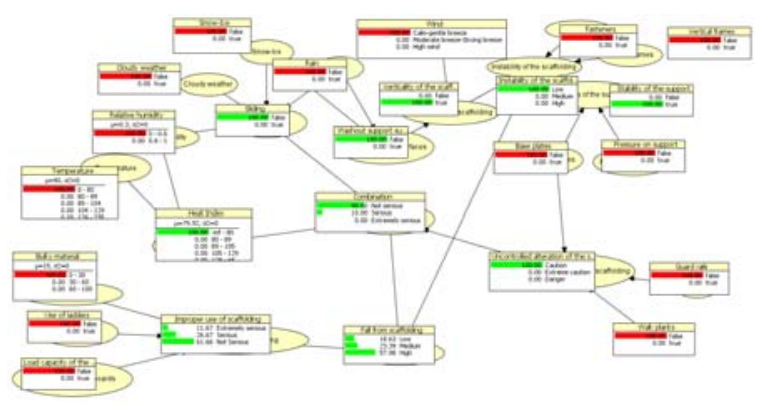

Fig. 17. Reference scenario in case of no adverse weather conditions and compliance to regulations.

In case outdoor weather favors heat stroke, due to high temperature (around $104^{\circ} \mathrm{F}$ ) and high relative humidity, even if wind is still calm, Fig. 18 shows that the expected risk of falling from heights is noticeably increased $\quad(<\mathrm{low}>=14.46 \%, \quad<$ medium $>=19.66 \%$, $<$ high $>=65.88 \%)$.

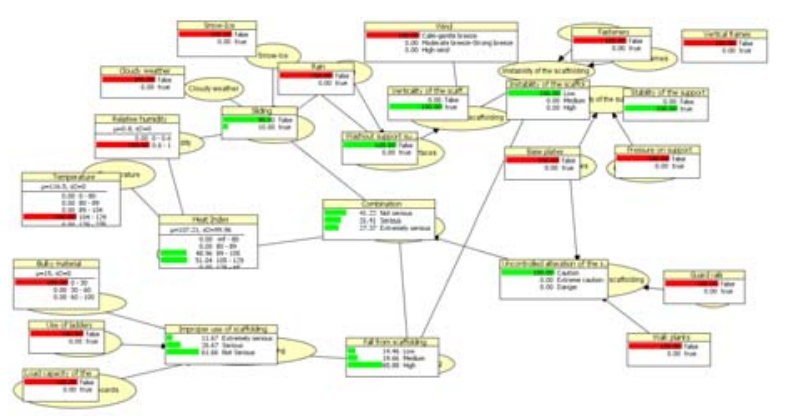

Fig. 18. The second scenario in case of weather conditions prone to heat stroke.

In the next scenario (see Fig. 19) we assume high wind intensity and the removal of fasteners, some of the vertical frames (weakening the whole steel frame of the scaffolding) and base plates under the scaffolding foot have been removed. Such a combination could lead to overall instability of the scaffolding and further increases the estimated risk level $(<$ low $>=7.44 \%$, $<$ medium $>=15.56 \%$, 


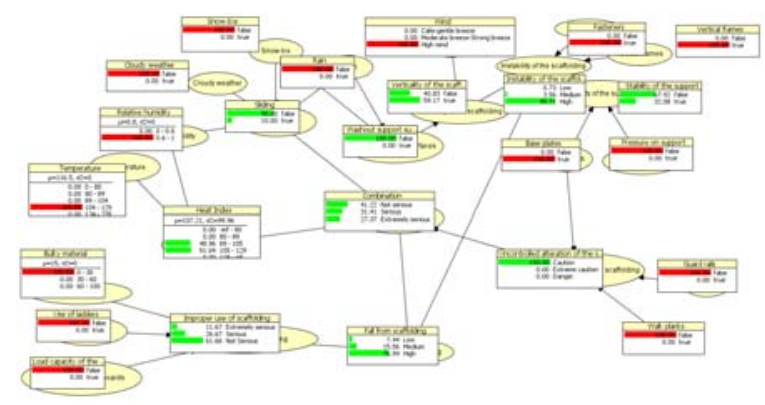

Fig. 19. The third scenario where scaffolding instability is more likely.

Finally, another far worse scenario where the presence of bulky materials on the scaffolding walk planks is assumed, along with the use of ladders by workers and the aforementioned material is so heavy to overcome the admissible threshold of load bearing capacity, Fig. 20 depicts the very high risk figure of falling from height $(<\mathrm{low}>=3.71 \%$, $<$ medium $>=7.49 \%,<$ high $>=88.80 \%)$.

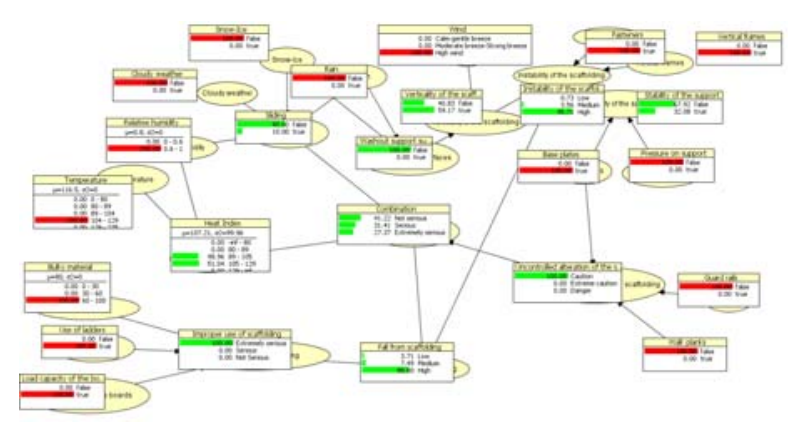

Fig. 20. The fourth scenario where even unfair workers behavior is assumed.

\section{Assessment on the network utilization for real- time safety management}

The network is ready to be implemented in a realtime application for safety management in construction sites, provided that sensors for inputs collection are designed and properly installed on site.

With respect to this aspect, Fig. 21 shows an assessment on the origin of input data.

Input data indicated as "Type A", refer to the weather conditions. Their variation could be immediately relieved by appropriate sensors and transmitted to the network in order to identify, in real time, possible risk scenarios.

Input data indicated as "Type B", directly refer to the effective configuration of the scaffolding and of its components (fasteners, vertical frames, base plates, guard-rails, walk planks). The low possibility of a continuous human control about the regular disposition of all the components of the scaffolding, suggests, also in this case, to employ appropriate sensors which are able to identify, in real time, any deviations from the planned configuration of all the components of the scaffolding.
Input data indicated as "Type C", provide information about static aspects of the scaffolding: the pressure on the supports and on the walk planks. The extreme difficulty to immediately recognize the overcoming of the admissible threshold of load bearing capacity, notwithstanding a great experience, suggests to employ a sensor set aim at monitoring, in real time, conditions prone to the overcoming of the load capacity of the scaffolding.

Input data indicated as "Type D" refer to workers behavior on the scaffolding. This kind of data could result from a human control (coordinator, scaffolding fitters, manager, etc.). Because workers behavior often dramatically increases the risk level of falling from height, a frequent control must be guaranteed. For this reason, this kind of input data could come from technological solutions which are aimed at obtaining constant automated monitoring of all the operative tasks on the scaffolding. Anyway, the network should record locations of the main resources of the specific construction site (workers, equipments, materials) highlighting the main risk scenarios of falling from height.

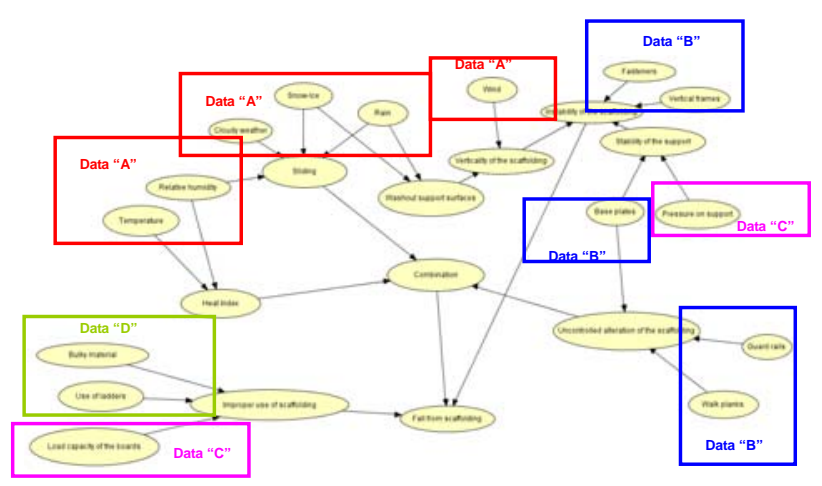

Fig. 21. The overall Bayesian network.

If the highlighted nodes of the overall network (see Fig. 21) collect data from sensors, all the other nodes will perform real time risk analysis on the basis of subjective knowledge which has been gathered through the elicitation process of the expert opinion described in the previous chapter.

\section{CONCLUSIONS AND FURTHER DEVELOPMENTS OF THE RESEARCH}

The present approach to health and safety management of construction sites owns strong intrinsic limits: every programmatic action whose scope is to minimize the probability of risky situations, notwithstanding its accuracy, must deal with the unforeseeable nature of all the construction dynamics. Our research focuses on the development of a new methodology for the identification and mitigation, in 
real time, of risk scenarios according to the real configuration of the construction site.

The methodological adopted scheme is based basically on two steps:

- one model for the identification and evaluation of significant risk situations (decision making support);

- the adoption of advanced sensors.

Hence data arriving from sensors would be evaluated, in real time, in order to identify relevant risk scenarios.

This paper reports the first result of our research project: the development of a Bayesian model focused on the risk scenario "fall from height" and its preliminary tests.

The future developments of the project will focus on the identification of the related sensor technology for real time control. The sensors will allow to gather data sets related to contextual conditions (e.g. Fig. 7 showed how weather conditions can increase the risk of fall from height: directly acting on the operators - heat stroke, and/or acting on the conditions of usability of the scaffolding - raining may cause any operator to slide. Even localization and monitoring of workers behavior have been found to be determinant for risk level estimation.

\section{ACKNOWLEDGEMENTS}

This research was funded by the Italian Ministry of Research and Education, PRIN Grant no. 2008P7T582_001.

\section{References}

1. INAIL (Istituto Nazionale per l'Assicuraizone contro gli Infortuni sul Lavoro), Banca dati INAIL, http://bancadati.inail.it/prevenzionale/

2. Eurostat, Health and safety at work statistics, 2011 http://epp.eurostat.ec.europa.eu/statistics_explained /index.php/Health_and_safety_at_work_statistics

3. Navon R., Kolton O., "Model for automated monitoring of fall hazards in building construction", Journal of Construction Engineering and Management vol. 132(7) pp. 733-740, 2006.

4. Council Directive 89/391/EEC of 12 June 1989 on the introduction of measures to encourage improvements in the safety and health of workers at work - Council Directive 92/57/EEC of 24 June 1992 on the implementation of minimum safety and health requirements at temporary or mobile construction sites (eighth individual Directive within the meaning of Article 16 of Directive 89/391/EEC

5. Aulin, R., Capone, P., "The role of health and safety coordinator in Sweden and Italy construction industry", 18th CIB W099 - World Building Congress, CIB publication 357, pp. 93-106, 2010.

6. Behm, M., "Linking construction fatalities to the design for construction safety concept", Safety Science, vol. 43(8), pp. 589-611,.2010.
7. Gambatese, J.A., Hinze, J., "Addressing construction worker safety in the design phase: Designing for construction worker safety", Automation in Construction, vol. 8(6), pp. 643-649, 1999.

8. Gangolells, M., Casals, M., Forcada, N., Roca, X., Fuertes, A., "Mitigating Construction Safety Risks using Prevention through Design", Journal of Safety Research vol. 41(2), pp. 107-122, 2010

9. BS OHSAS 18001, Occupational Health and Safety, 2005

10. Swedberg, C., "BP Refinery Uses RFID for Evacuation System", RFID Journal, available on line at: www.rfidjournal.com/article/articleview/2389/1/1/ 2006.

11. Zetik, R., Sachs, J.,Thoma, Reiner S., "UWB shortrange radar sensing", IEEE Instrumentation and Measurement Magazine, v 10, n 2, p.39-45, 2007.

12. Caldas, C. H., Haas, C. T., Liapi, K. A., Teizer, J., "Modeling job sites in real time to improve safety during equipment operation", Proceedings of SPIE The international Society for Optical Engineering, vol. 6174(II), p. 61743k, 2006.

13. Abderrahim M., Garcia E., Diez R., Balaguer C., A mechatronics security system for the construction site, Automation in Construction, vol. 14(4) pp. 460466, 2005.

14. Victores J.G., Martinez S., Jardon A., Balaguer C., "Robot-aided tunnel inspection and maintenance system by vision and proximity sensor integration", Automation in Construction, vol. 20(5) pp. 629-636, 2011.

15. Tantisevi K., Akinci B., "Automated generation of workspace requirements of mobile crane operations to support conflict detection", Automation in Construction, vol. 16(3) pp. 262-276, 2007.

16. Kim J.H., Lee U.K, Cho H., Kang K.I., "Development of a mobile safety monitoring system for construction sites", Automation in Construction, vol. 18(3) pp. 258-264, 2009.

17. Cho Y.K., Youn J.H., Martinez D., "Error modeling for an untethered ultra-wideband system for construction indoor asset tracking", Automation in Construction vol. 19(1) pp.43-54, 2010.

18. Saidi K. S., Teizer J., Franaszek M., Lytle A. M., "Static and dynamic performance evaluation of a commercially-available ultra wideband tracking system", Automation in Construction. vol. 20(5) pp. 519-530, 2011.

19. Lu W., Huang G.Q., Li H., "Scenarios for applying rfid technology in construction project management", Automation in Construction vol. 20(2) pp. 101-106, 2011.

20. Pearl J., "Probabilistic reasoning in intelligent systems: networks of plausible inference", Morgan Kaufman, 1988.

21. Kerzner H., "Project Management. A systems approach to Planning, Scheduling and Controlling", John Wiley \&Sons Inc., eighth edition, pp. 396-402, 2003.

22. Vose D., "Risk Analysis. A quantitative guide", John Wiley \&Sons Inc., third edition pp. 401-422, 2008. 